\title{
The Dynamics Theorem for properly embedded minimal surfaces
}

\author{
William H. Meeks III* \\ Joaquín Pérez \\ Antonio Ros ${ }^{\dagger}$
}

June 1,2021

\begin{abstract}
In this paper we prove two theorems. The first one is a structure result that describes the extrinsic geometry of an embedded surface with constant mean curvature (possibly zero) in a homogeneously regular Riemannian three-manifold, in any small neighborhood of a point of large almost-maximal curvature. We next apply this theorem and the Quadratic Curvature Decay Theorem in [14 to deduce compactness, descriptive and dynamics-type results concerning the space $D(M)$ of non-flat limits under dilations of any given properly embedded minimal surface $M$ in $\mathbb{R}^{3}$.
\end{abstract}

Mathematics Subject Classification: Primary 53A10, Secondary 49Q05, 53C42

Key words and phrases: Minimal surface, constant mean curvature, stability, curvature estimates, finite total curvature, minimal lamination, dynamics theorem.

\section{Introduction.}

In this paper we will describe the local extrinsic geometry of a complete embedded surface $M$ of constant mean curvature around a point of large Gaussian curvature in a homogeneously regular Riemannian three-manifold (see Theorem 1.1). We then obtain some consequences among which we highlight the Dynamics Theorem (Theorem 1.3) concerning the space $D(M)$ of non-flat limits under a divergent sequence of dilations of any given properly embedded minimal surface $M$ in $\mathbb{R}^{3}$; by a dilation we mean any diffeomorphism of $\mathbb{R}^{3}$ into itself given by composition of a translation with a homothety. An important consequence of the Dynamics Theorem is that every properly embedded minimal surface in $\mathbb{R}^{3}$ with infinite total curvature has a surprising amount of internal dynamical periodicity; see Theorem 1.3 and Proposition 6.1 below for this interpretive consequence.

\footnotetext{
${ }^{*}$ This material is based upon work for the NSF under Award No. DMS - 1309236. Any opinions, findings, and conclusions or recommendations expressed in this publication are those of the authors and do not necessarily reflect the views of the NSF.

${ }^{\dagger}$ The second and third authors were supported in part by the MEC/FEDER grant no. MTM2011-22547, and by the regional J. Andalucía grant no. P06-FQM-01642.
} 
In order to state the main results, it is worth setting some specific notation to be used throughout the paper. Given a Riemannian three-manifold $N$ and a point $p \in N$, we denote by $B_{N}(p, r), \bar{B}_{N}(p, r), S_{N}^{2}(p, r)$ the open metric ball of center $p$ and radius $r>0$, its closure and boundary sphere, respectively. In the case $N=\mathbb{R}^{3}$, we use the notation $\mathbb{B}(p, r)=B_{\mathbb{R}^{3}}(p, r)$, $\mathbb{S}^{2}(p, r)=S_{\mathbb{R}^{3}}^{2}(p, r)$ and $\mathbb{B}(r)=\mathbb{B}(\overrightarrow{0}, r), \mathbb{S}^{2}(r)=\mathbb{S}^{2}(\overrightarrow{0}, r)$, where $\overrightarrow{0}=(0,0,0)$. For a surface $M$ embedded in $N$, we denote by $\left|\sigma_{M}\right|$ the norm of the second fundamental form of $M$. Finally, for a surface $M \subset \mathbb{R}^{3}, K_{M}$ denotes its Gaussian curvature function. We will call a Riemannian three-manifold $N$ homogeneously regular if there exists an $\varepsilon>0$ such that $\varepsilon$-balls in $N$ are uniformly close to $\varepsilon$-balls in $\mathbb{R}^{3}$ in the $C^{2}$-norm. In particular, if $N$ is compact, then $N$ is homogeneously regular.

Theorem 1.1 (Local Picture on the Scale of Curvature) Suppose $M$ is a complete, embedded, constant mean curvature surface (here we include minimal surfaces as being those with constant mean curvature zero) with unbounded second fundamental form in a homogeneously regular three-manifold $N$. Then, there exists a sequence of points $p_{n} \in M$ and positive numbers $\varepsilon_{n} \rightarrow 0$, such that the following statements hold.

1. For all $n$, the component $M_{n}$ of $\bar{B}_{N}\left(p_{n}, \varepsilon_{n}\right) \cap M$ that contains $p_{n}$ is compact with boundary $\partial M_{n} \subset \partial B_{N}\left(p_{n}, \varepsilon_{n}\right)$.

2. Let $\lambda_{n}=\left|\sigma_{M_{n}}\left(p_{n}\right)\right|$. Then, $\lim _{n \rightarrow \infty} \varepsilon_{n} \lambda_{n}=\infty$ and $\frac{\left|\sigma_{M_{n}}\right|}{\lambda_{n}} \leq 1+\frac{1}{n}$ on $M_{n}$. .

3. The metric balls $\lambda_{n} B_{N}\left(p_{n}, \varepsilon_{n}\right)$ of radius $\lambda_{n} \varepsilon_{n}$ converge uniformly to $\mathbb{R}^{3}$ with its usual metric (so that we identify $p_{n}$ with $\overrightarrow{0}$ for all $n$ ), and, for any $k \in \mathbb{N}$, the surfaces $\lambda_{n} M_{n}$ converge $C^{k}$ on compact subsets of $\mathbb{R}^{3}$ and with multiplicity one to a connected, properly embedded minimal surface $M_{\infty}$ in $\mathbb{R}^{3}$ with $\overrightarrow{0} \in M_{\infty},\left|\sigma_{M_{\infty}}\right| \leq 1$ on $M_{\infty}$ and $\left|\sigma_{M_{\infty}}\right|(\overrightarrow{0})=1$.

Every complete, embedded minimal surface in $\mathbb{R}^{3}$ with bounded curvature is properly embedded, see Meeks and Rosenberg [20. The key idea in the proof of Theorem 1.1 is to exploit this fact, together with a careful blow-up argument. Note that a direct consequence of Theorem 1.1 is that every complete embedded surface with constant mean curvature in $\mathbb{R}^{3}$ which is not properly embedded, has natural limits under a sequence of dilations, which are properly embedded non-flat minimal surfaces.

Theorem 1.1 together with the Local Removable Singularity Theorem in [14] can be used to understand the structure of the collection of limits of a non-flat, properly embedded minimal surface in $\mathbb{R}^{3}$ under any divergent sequence of dilations, which is the purpose of the next theorem. In order to clarify its statement, we need some definitions.

Definition 1.2 Let $M \subset \mathbb{R}^{3}$ be a non-flat, properly embedded minimal surface. Then:

1. $M$ is periodic if it is invariant under a non-trivial translation or screw motion symmetry. 
2. $M$ is quasi-translation-periodic if there exists a divergent sequence $\left\{p_{n}\right\}_{n} \subset \mathbb{R}^{3}$ such that $\left\{M-p_{n}\right\}_{n}$ converges $C^{2}$ on compact subsets of $\mathbb{R}^{3}$ to $M$; note that every periodic surface is also quasi-translation-periodic, even in the case the surface is invariant under a screw motion symmetry.

3. $M$ is quasi-dilation-periodic if there exists a sequence $\left\{\lambda_{n}\right\}_{n} \subset \mathbb{R}^{+}$and a divergent sequence $\left\{p_{n}\right\}_{n} \subset \mathbb{R}^{3}$ such that the sequence $\left\{\lambda_{n}\left(M-p_{n}\right)\right\}_{n}$ of dilations of $M$ converges in a $C^{2}$ manner on compact subsets of $\mathbb{R}^{3}$ to $M$. Since $M$ is not flat, then it is not stable and, it can be proved that the convergence of such a sequence $\left\{h_{n}\left(M-p_{n}\right)\right\}_{n}$ to $M$ has multiplicity one (see Lemma 4.1 below for a similar result about multiplicity one convergence).

4. Let $D(M)$ be the set of non-flat, properly embedded minimal surfaces in $\mathbb{R}^{3}$ which are obtained as $C^{2}$-limits of a divergent sequence $\left\{\lambda_{n}\left(M-p_{n}\right)\right\}_{n}$ of dilations of $M$ (i.e., the translational part $\left\{p_{n}\right\}_{n}$ of the dilations diverges). A non-empty subset $\Delta \subset D(M)$ is called $D$-invariant, if for any $\Sigma \in \Delta$, then $D(\Sigma) \subset \Delta$. A $D$-invariant subset $\Delta \subset D(M)$ is called a minimal $D$-invariant set, if it contains no proper, non-empty $D$-invariant subsets. We say that $\Sigma \in D(M)$ is a minimal element, if $\Sigma$ is an element of a minimal $D$-invariant subset of $D(M)$.

Theorem 1.3 (Dynamics Theorem) Let $M \subset \mathbb{R}^{3}$ be a properly embedded, non-flat minimal surface. Then, $D(M)=\varnothing$ if and only if $M$ has finite total curvature. Now assume that $M$ has infinite total curvature, and consider $D(M)$ endowed with the topology of $C^{k}$-convergence on compact sets of $\mathbb{R}^{3}$ for all $k$. Then:

1. $D_{1}(M)=\left\{\Sigma \in D(M)|\overrightarrow{0} \in \Sigma,| K_{\Sigma}|\leq 1,| K_{\Sigma} \mid(\overrightarrow{0})=1\right\}$ is a non-empty compact subspace of $D(M)$.

2. For any $\Sigma \in D(M), D(\Sigma)$ is a closed D-invariant set of $D(M)$. If $\Delta \subset D(M)$ is a $D$-invariant set, then its closure $\bar{\Delta}$ in $D(M)$ is also D-invariant.

3. Suppose that $\Delta \subset D(M)$ is a non-empty minimal $D$-invariant set which does not consist of exactly one surface of finite total curvature. If $\Sigma \in \Delta$, then $D(\Sigma)=\Delta$ and the closure in $D(M)$ of the path connected subspace $\left\{\lambda(\Sigma-p) \mid p \in \mathbb{R}^{3}, \lambda>0\right\}$ of all dilations of $\Sigma$ equals $\Delta$. In particular, any minimal $D$-invariant set is connected and closed in $D(M)$.

4. Every non-empty D-invariant subset of $D(M)$ contains minimal elements. In particular, since $D(M)$ is $D$-invariant, then $D(M)$ always contains some minimal element.

5. Let $\Delta \subset D(M)$ be a non-empty $D$-invariant subset. If no $\Sigma \in \Delta$ has finite total curvature, then $\Delta_{1}=\left\{\Sigma \in \Delta|\overrightarrow{0} \in \Sigma,| K_{\Sigma}|\leq 1,| K_{\Sigma} \mid(\overrightarrow{0})=1\right\}$ contains a minimal element $\Sigma^{\prime}$, and every such surface $\Sigma^{\prime}$ satisfies that $\Sigma^{\prime} \in D\left(\Sigma^{\prime}\right)$ (in particular, $\Sigma^{\prime}$ is a quasi-dilation-periodic surface of bounded curvature). 
6. If a minimal element $\Sigma$ of $D(M)$ has finite genus, then either $\Sigma$ has finite total curvature, $\Sigma$ is a helicoid, or $\Sigma$ is a Riemann minimal example.

7. If a minimal element $\Sigma$ of $D(M)$ has more than one end, then every middle end of $\Sigma$ is smoothly asymptotic to the end of a plane or catenoid.

\section{Preliminaries.}

When proving the results stated in the introduction, we will make use of three results from our previous paper [14]. For the reader's convenience, we collect here these results and the definitions necessary in order to state them.

Definition 2.1 A codimension one lamination $\mathcal{L}$ of a Riemannian three-manifold $N$ is the union of a collection of pairwise disjoint, connected, injectively immersed surfaces called leaves of $\mathcal{L}$, with a certain local product structure. More precisely, it is a pair $(\mathcal{L}, \mathcal{A})$ satisfying:

1. $\mathcal{L}$ is a closed subset of $N$;

2. $\mathcal{A}=\left\{\varphi_{\beta}: \mathbb{D} \times(0,1) \rightarrow U_{\beta}\right\}_{\beta}$ is an atlas of coordinate charts of $N$ (here $\mathbb{D}$ is the open unit disk in $\mathbb{R}^{2},(0,1)$ is the open unit interval and $U_{\beta}$ is an open subset of $N$ ); note that although $N$ is assumed to be smooth, we only require that the regularity of the atlas (i.e., that of its change of coordinates) is of class $C^{0}$, i.e., $\mathcal{A}$ is an atlas for the topological structure of $N$.

3. For each $\beta$, there exists a closed subset $C_{\beta}$ of $(0,1)$ such that $\varphi_{\beta}^{-1}\left(U_{\beta} \cap \mathcal{L}\right)=\mathbb{D} \times C_{\beta}$.

A leaf $L$ of $\mathcal{L}$ is called a limit leaf if for some (or every) point $p \in L$, there exists a coordinate chart $\varphi_{\beta}: \mathbb{D} \times(0,1) \rightarrow U_{\beta}$ as in Definition 2.1 such that $p \in U_{\beta}$ and $\varphi_{\beta}^{-1}(p)=(x, t)$ with $t$ belonging to the accumulation set of $C_{\beta}$.

We will simply denote laminations by $\mathcal{L}$, omitting the charts $\varphi_{\beta}$ in $\mathcal{A}$. Every lamination $\mathcal{L}$ naturally decomposes into a collection of disjoint, connected topological surfaces (locally given by $\varphi_{\beta}(\mathbb{D} \times\{t\}), t \in C_{\beta}$, with the notation above), called the leaves of $\mathcal{L}$. As usual, the regularity of $\mathcal{L}$ requires the corresponding regularity on the change of coordinate charts $\varphi_{\beta}$. A lamination $\mathcal{L}$ of $N$ is called a foliation of $N$ if $\mathcal{L}=N$. A lamination $\mathcal{L}$ of $N$ is said to be a minimal lamination if all its leaves are smooth with zero mean curvature. Since the leaves of $\mathcal{L}$ are pairwise disjoint, we can consider the second fundamental form $\left|\sigma_{\mathcal{L}}\right|$ of $\mathcal{L}$, which is the function defined at any point $p \in \mathcal{L}$ as $\left|\sigma_{L}\right|(p)$, where $L$ is the unique leaf of $\mathcal{L}$ passing through $p$.

There are three key results that we will need from [14], and which we list below for the readers convenience. 
Theorem 2.2 (Local Removable Singularity Theorem [14]) A minimal lamination $\mathcal{L}$ of a punctured ball $B_{N}(p, r)-\{p\}$ in a Riemannian three-manifold $N$ extends to a minimal lamination of $B_{N}(p, r)$ if and only if there exists a positive constant $C$ such that $\left|\sigma_{\mathcal{L}}\right| d_{N}(p, \cdot) \leq C$ in some subball.

Definition 2.3 In the sequel, we will denote by $R=\sqrt{x_{1}^{2}+x_{2}^{2}+x_{3}^{2}}$ the radial distance to the origin $\overrightarrow{0} \in \mathbb{R}^{3}$. A surface $M \subset \mathbb{R}^{3}$ has quadratic decay of curvature if there exists $C>0$ such that $\left|K_{M}\right| R^{2} \leq C$ on $M$. Analogously, a minimal lamination $\mathcal{L}$ of $\mathbb{R}^{3}$ or of $\mathbb{R}^{3}-\{\overrightarrow{0}\}$ has quadratic decay of curvature if $\left|K_{\mathcal{L}}\right| R^{2}$ is bounded on $\mathcal{L}$, where $\left|K_{\mathcal{L}}\right|$ is the function that associates to each point $p \in \mathcal{L}$ the absolute Gaussian curvature of the unique leaf of $\mathcal{L}$ passing through $p$.

Theorem 2.4 (Quadratic Curvature Decay Theorem [14]) A complete, embedded minimal surface in $\mathbb{R}^{3}$ with compact boundary (possibly empty) has quadratic decay of curvature if and only if it has finite total curvature. In particular, a complete, connected embedded minimal surface $M \subset \mathbb{R}^{3}$ with compact boundary and quadratic decay of curvature is properly embedded in $\mathbb{R}^{3}$.

Proposition 2.5 (Corollary 6.3 in [14]) Let $\mathcal{L}$ be a non-flat minimal lamination of $\mathbb{R}^{3}-\{\overrightarrow{0}\}$. If $\mathcal{L}$ has quadratic decay of curvature, then $\mathcal{L}$ consists of a single leaf, which extends across $\overrightarrow{0}$ to a properly embedded minimal surface with finite total curvature in $\mathbb{R}^{3}$.

\section{Proof of the Local Picture Theorem on the Scale of Curvature.}

The proof of Theorem 1.1 stated in the introduction uses a blow-up technique, where the scaling factors are given by the norm of the second fundamental form of $M$ at points of large almostmaximal curvature, a concept which we develop below. After the blowing-up process, we will find a limit which is a complete minimal surface with bounded Gaussian curvature in $\mathbb{R}^{3}$, conditions which are known to imply properness for the limit [20]. This properness property will lead to the conclusions of Theorem 1.1 .

Proof of Theorem 1.1. Since $N$ is homogeneously regular, after a fixed constant scaling of the metric of $N$ we may assume that the injectivity radius of $N$ is greater than 1 . The first step in the proof is to obtain special points $p_{n}^{\prime} \in M$, called blow-up points or points of almost-maximal curvature. First consider an arbitrary sequence of points $q_{n} \in M$ such that $\left|\sigma_{M}\right|\left(q_{n}\right) \geq n$, which exists since $\left|\sigma_{M}\right|$ is unbounded. Let $p_{n}^{\prime} \in B_{M}\left(q_{n}, 1\right)$ be a maximum of the function $h_{n}=\left|\sigma_{M}\right| d_{M}\left(\cdot, \partial B_{M}\left(q_{n}, 1\right)\right)$, where $d_{M}$ stands for the intrinsic distance on $M$. We define $\lambda_{n}^{\prime}=\left|\sigma_{M}\right|\left(p_{n}^{\prime}\right)$. Note that for each $n \in \mathbb{N}$,

$$
\lambda_{n}^{\prime} \geq \lambda_{n}^{\prime} d_{M}\left(p_{n}^{\prime}, \partial B_{M}\left(q_{n}, 1\right)\right)=h_{n}\left(p_{n}^{\prime}\right) \geq h_{n}\left(q_{n}\right)=\left|\sigma_{M}\right|\left(q_{n}\right) \geq n .
$$

Fix $t>0$. Since $\lambda_{n}^{\prime} \rightarrow \infty$ as $n \rightarrow \infty$, the sequence $\left\{\lambda_{n}^{\prime} B_{N}\left(p_{n}^{\prime}, \frac{t}{\lambda_{n}^{\prime}}\right)\right\}_{n}$ converges to the open ball $\mathbb{B}(t)$ of $\mathbb{R}^{3}$ with its usual metric, where we have used geodesic coordinates in $N$ centered 
at $p_{n}^{\prime}$ and identified $p_{n}^{\prime}$ with $\overrightarrow{0}$. Similarly, we can consider $\left\{\lambda_{n}^{\prime} B_{M}\left(p_{n}^{\prime}, \frac{t}{\lambda_{n}^{\prime}}\right)\right\}_{n}$ to be a sequence of embedded, constant mean curvature surfaces with non-empty topological boundary, all passing through $\overrightarrow{0}$ with norm of their second fundamental forms 1 at this point. We claim that the sequence of second fundamental forms of $\lambda_{n}^{\prime} B_{M}\left(p_{n}^{\prime}, \frac{t}{\lambda_{n}^{\prime}}\right)$ is uniformly bounded. To see this, pick a point $z_{n} \in B_{M}\left(p_{n}^{\prime}, \frac{t}{\lambda_{n}^{\prime}}\right)$. Note that for $n$ large enough, $z_{n}$ lies in $B_{M}\left(q_{n}, 1\right)$. Then,

$$
\frac{\left|\sigma_{M}\right|\left(z_{n}\right)}{\lambda_{n}^{\prime}}=\frac{h_{n}\left(z_{n}\right)}{\lambda_{n}^{\prime} d_{M}\left(z_{n}, \partial B_{M}\left(q_{n}, 1\right)\right)} \leq \frac{d_{M}\left(p_{n}^{\prime}, \partial B_{M}\left(q_{n}, 1\right)\right)}{d_{M}\left(z_{n}, \partial B_{M}\left(q_{n}, 1\right)\right)} .
$$

By the triangle inequality, $d_{M}\left(p_{n}^{\prime}, \partial B_{M}\left(q_{n}, 1\right)\right) \leq \frac{t}{\lambda_{n}^{\prime}}+d_{M}\left(z_{n}, \partial B_{M}\left(q_{n}, 1\right)\right)$, and so,

$$
\begin{aligned}
& \frac{d_{M}\left(p_{n}^{\prime}, \partial B_{M}\left(q_{n}, 1\right)\right)}{d_{M}\left(z_{n}, \partial B_{M}\left(q_{n}, 1\right)\right)} \leq 1+\frac{t}{\lambda_{n}^{\prime} d_{M}\left(z_{n}, \partial B_{M}\left(q_{n}, 1\right)\right)} \\
& \leq 1+\frac{t}{\lambda_{n}^{\prime}\left(d_{M}\left(p_{n}^{\prime}, \partial B_{M}\left(q_{n}, 1\right)\right)-\frac{t}{\lambda_{n}^{\prime}}\right)} \leq 1+\frac{t}{n-t},
\end{aligned}
$$

which tends to 1 as $n \rightarrow \infty$.

It follows that after extracting a subsequence, the surfaces $\lambda_{n}^{\prime} B_{M}\left(p_{n}^{\prime}, \frac{t}{\lambda_{n}^{\prime}}\right)$ converge (possibly with non-constant integer or infinite multiplicity) to an embedded minimal surface $M_{\infty}(t)$ contained in $\mathbb{B}(t)$ with bounded Gaussian curvature, that passes through $\overrightarrow{0}$ and with norm of its second fundamental form 1 at the origin; note that the topological boundary $\partial M_{\infty}(t)$ of $M_{\infty}(t)$ need not be either smooth or contained in $\mathbb{S}^{2}(t)$. Consider the surface $M_{\infty}(1)$ together with the surfaces $\lambda_{n}^{\prime} B_{M}\left(p_{n}^{\prime}, \frac{1}{\lambda_{n}^{\prime}}\right)$ that converge to it (after passing to a subsequence). Note that $M_{\infty}(1)$ is contained in $M_{\infty}=\bigcup_{t \geq 1} M_{\infty}(t)$, which is a complete, injectively immersed minimal surface in $\mathbb{R}^{3}$, with $\overrightarrow{0} \in M_{\infty}$ and $\left|\sigma_{M_{\infty}}\right|(\overrightarrow{0})=1$. By construction, $M_{\infty}$ has bounded Gaussian curvature, so it is properly embedded in $\mathbb{R}^{3}[20$.

The next result describes how the surfaces $\lambda_{n}^{\prime} B_{M}\left(p_{n}^{\prime}, \frac{t}{\lambda_{n}^{\prime}}\right)$ that give rise to the limit $M_{\infty}(t)$ sit nicely in space with respect to the surface $M_{\infty}(t+1)$.

Lemma 3.1 Given $t>0$, there exist $k \in \mathbb{N}$ such that if $n \geq k$, then $\lambda_{n}^{\prime} B_{M}\left(p_{n}^{\prime}, \frac{t}{\lambda_{n}^{\prime}}\right)$ is contained in a small regular neighborhood of $M_{\infty}(t+1)$ in $\mathbb{R}^{3}$. Furthermore, $\lambda_{n}^{\prime} B_{M}\left(p_{n}^{\prime}, \frac{t}{\lambda_{n}^{\prime}}\right)$ is a small normal graph over its projection to $M_{\infty}(t+1)$.

Proof. Let $\pi: \widetilde{M}_{\infty} \rightarrow M_{\infty}$ be the universal cover of $M_{\infty}$. Choose a point $\widetilde{x} \in \pi^{-1}(\{\overrightarrow{0}\})$. Since $M_{\infty}$ is not flat, then $\widetilde{M}_{\infty}$ is not stable [6, 8, 24] and thus, there exists $R>0$ such that the intrinsic ball $B_{\widetilde{M}_{\infty}}(\widetilde{x}, R)$ centered at $\widetilde{x}$ with radius $R$ is unstable.

Choose $t>0$. Since the closure $\bar{M}_{\infty}(t)$ of $M_{\infty}(t)$ is compact (because $M_{\infty}(t) \subset \mathbb{B}(t)$ ), then there exists $\delta_{t}>0$ such that $\bar{M}_{\infty}(t)$ admits a regular neighborhood $U(t) \subset \mathbb{R}^{3}$ of radius 
$\delta_{t}>0$; in particular, $U(t)$ is diffeomorphic to $\bar{M}_{\infty}(t) \times\left(-\delta_{t}, \delta_{t}\right)$ and we have a related normal projection $\Pi_{t}: U(t) \rightarrow \bar{M}_{\infty}(t)$. Let $\pi_{t}: \widetilde{M}_{\infty}(t) \rightarrow \bar{M}_{\infty}(t)$ be the universal cover of $\bar{M}_{\infty}(t)$ and let $\pi_{t} \times \operatorname{Id}: \widetilde{U}(t) \equiv \widetilde{M}_{\infty}(t) \times\left(-\delta_{t}, \delta_{t}\right) \rightarrow \bar{M}_{\infty}(t) \times\left(-\delta_{t}, \delta_{t}\right)$ be the universal cover of $U(t)$, each one endowed with the pulled back metric. Therefore, we also have a normal projection $\widetilde{\Pi}_{t}: \widetilde{U}(t) \rightarrow \widetilde{M}_{\infty}(t)$ such that $\pi_{t} \circ \widetilde{\Pi}_{t}=\Pi_{t} \circ\left(\pi_{t} \times \mathrm{Id}\right)$.

Since the sequence $\left\{\lambda_{n}^{\prime} B_{M}\left(p_{n}^{\prime}, \frac{t}{\lambda_{n}^{\prime}}\right)\right\}_{n}$ converges to $M_{\infty}(t)$ as $n \rightarrow \infty$, there exists $n_{0}=$ $n_{0}(t) \in \mathbb{N}$ such that for every $n \geq n_{0}$, we have $\lambda_{n}^{\prime} B_{M}\left(p_{n}^{\prime}, \frac{t}{\lambda_{n}^{\prime}}\right) \subset U(t+1)$, which clearly implies the first sentence in the statement of Lemma 3.1. To prove the second statement, we argue by contradiction. Suppose that for some $t>0$, there exists a sequence of integer numbers $n(m) \geq n_{0}(t)$ tending to $\infty$ such that for each $m, \lambda_{n(m)}^{\prime} B_{M}\left(p_{n(m)}^{\prime}, \frac{t}{\lambda_{n(m)}^{\prime}}\right)$ fails to be a normal graph over its projection to $M_{\infty}(t+1)$. To keep the notation simple, we will relabel $n(m)$ as $n$. This means that for each $n \geq n_{0}(t)$, there exist two distinct points $q_{n}(1), q_{n}(2) \in \lambda_{n}^{\prime} B_{M}\left(p_{n}^{\prime}, \frac{t}{\lambda_{n}^{\prime}}\right)$ such that $\Pi_{t+1}\left(q_{n}(1)\right)=\Pi_{t+1}\left(q_{n}(2)\right)$. As the sequence $\left\{\Pi_{t+1}\left(q_{n}(1)\right)\right\}_{n \geq n_{0}(t)}$ lies in the compact set $\bar{M}_{\infty}(t+1)$, after extracting a subsequence we may assume that the $\Pi_{t+1}\left(q_{n}(1)\right)=\Pi_{t+1}\left(q_{n}(2)\right)$ converge as $n \rightarrow \infty$ to a point $q_{\infty} \in \bar{M}_{\infty}(t)$. Therefore, there exists some $\varepsilon=\varepsilon(t)>0$ small such that for each $n \geq n_{0}(t), \lambda_{n}^{\prime} B_{M}\left(p_{n}^{\prime}, \frac{t+1}{\lambda_{n}^{\prime}}\right)$ contains two disjoint disks $D_{1}(n), D_{2}(n)$ in such a way that

$$
\left.\left(\Pi_{t+1}\right)\right|_{D_{i}(n)}: D_{i}(n) \rightarrow B_{M_{\infty}}\left(q_{\infty}, \varepsilon\right) \quad \text { is a diffeomorphism, } i=1,2,
$$

where $B_{M_{\infty}}\left(q_{\infty}, \varepsilon\right) \subset M_{\infty}(t+1)$ denotes the geodesic disk in $M_{\infty}$ centered at $q_{\infty}$ with radius $\varepsilon$. Consider the universal covering

$$
\pi=\pi_{t+R+2}: \widetilde{M}_{\infty}(t+R+2) \rightarrow \bar{M}_{\infty}(t+R+2) .
$$

Choose a point $\widetilde{q}_{\infty} \in \pi^{-1}\left(\left\{q_{\infty}\right\}\right) \subset \widetilde{M}_{\infty}(t+R+2)$ such that the distance from $\widetilde{x}$ to $\widetilde{q}$ is less than or equal to $t$ (this can be done since $q_{\infty} \in \bar{B}_{M_{\infty}}(\overrightarrow{0}, t)$ ). We will find the desired contradiction by constructing a positive Jacobi function on the closed intrinsic ball $\bar{B}_{\widetilde{M}_{\infty}(t+R+2)}\left(\widetilde{q}_{\infty}, t+R\right)$, which is impossible since this last closed ball contains the unstable domain $B_{\widetilde{M}_{\infty}}(\widetilde{x}, R)$ by the triangle inequality.

Take $n_{0}=n_{0}(t)$ large such that for all $n \geq n_{0}, \lambda_{n}^{\prime} \bar{B}_{M}\left(p_{n}^{\prime}, \frac{t+R+1}{\lambda_{n}^{\prime}}\right)$ lies in $U(t+R+2)$. Hence, we can lift $\lambda_{n}^{\prime} \bar{B}_{M}\left(p_{n}^{\prime}, \frac{t+R+1}{\lambda_{n}^{\prime}}\right)$ to $\widetilde{U}(t+R+2)$ via the covering $\pi \times \operatorname{Id}$. Note that

$$
V_{n}(t, R):=(\pi \times \mathrm{Id})^{-1}\left[\lambda_{n}^{\prime} B_{M}\left(p_{n}^{\prime}, \frac{t+R+1}{\lambda_{n}^{\prime}}\right)\right]
$$

is a possibly disconnected, non-compact surface in $\widetilde{U}(t+R+2)$. As $B_{M_{\infty}}\left(q_{\infty}, \varepsilon\right) \subset M_{\infty}(t+1)$ is a disk and $\pi$ is a Riemannian covering, then $B_{M_{\infty}}\left(q_{\infty}, \varepsilon\right)$ lifts to the geodesic disk $B_{\widetilde{M}_{\infty}(t+R+2)}\left(\widetilde{q}_{\infty}, \varepsilon\right)$ in $\widetilde{M}_{\infty}(t+R+2)$ with center $\widetilde{q}_{\infty}$ and radius $\varepsilon$. Using (3), we can lift $D_{1}(n), D_{2}(n)$ to small disks $\widetilde{D}_{1}(n), \widetilde{D}_{2}(n) \subset V_{n}(t, R)$ such that

$$
\left.\left(\widetilde{\Pi}_{t+R+2}\right)\right|_{\widetilde{D}_{i}(n)}: \widetilde{D}_{i}(n) \rightarrow B_{\widetilde{M}_{\infty}(t+R+2)}\left(\widetilde{q}_{\infty}, \varepsilon\right) \quad \text { is a diffeomorphism, } i=1,2 .
$$


As the closed intrinsic metric ball $\bar{B}_{\widetilde{M}_{\infty}(t+R+2)}\left(\widetilde{q}_{\infty}, R\right)$ is compact and the sequence of geodesic disks $\left\{\lambda_{n}^{\prime} B_{M}\left(p_{n}^{\prime}, \frac{t+R+1}{\lambda_{n}^{\prime}}\right)\right\}_{n}$ converges smoothly to $M_{\infty}(t+R+1) \subset M_{\infty}(t+R+2)$, then given $\mu>0$ small there exists $n_{1}=n_{1}(t, \mu) \in \mathbb{N}$ (we may assume $n_{1} \geq n_{0}(t)$ ) such that for every $n \geq n_{1}$, the normal projection $\widetilde{\Pi}_{t+R+2}$ restricts to $V_{n}(t, R)$ as a $\mu$-quasi-isometry, in the sense that the ratio between the length of tangent vectors at points in $V_{n}(t, R)$ and their images through the differential of $\widetilde{\Pi}_{t+R+2}$ lies in the range $[1-\mu, 1+\mu]$. In particular, every radial geodesic arc $\gamma \subset \bar{B}_{\widetilde{M}_{\infty}(t+R+2)}\left(\widetilde{q}_{\infty}, t+R\right)$ starting at $\widetilde{q}_{\infty}$ and ending at $\partial \bar{B}_{\widetilde{M}_{\infty}(t+R+2)}\left(\widetilde{q}_{\infty}, t+R\right)$ can be uniquely lifted to a pair of disjoint embedded $\operatorname{arcs} \gamma_{1}(n), \gamma_{n}(n) \subset V_{n}(t, R)$ starting at the points $\widehat{q}_{i}(\infty):=\left(\left.\widetilde{\Pi}_{t+R+2}\right|_{\widetilde{D}_{i}(n)}\right)^{-1}\left(\widetilde{q}_{\infty}\right) \in \widetilde{D}_{i}(n)$, and these arcs have length less than or equal to $t+R+1$, for $i=1,2$. When $\gamma$ varies in the set of radial geodesic arcs starting at $\widetilde{q}_{\infty}$ and ending at $\partial \bar{B}_{\widetilde{M}_{\infty}(t+R+2)}\left(\widetilde{q}_{\infty}, t+R\right)$, the union of the related lifted arcs $\gamma_{1}(n), \gamma_{2}(n)$ give rise to closed, disjoint topological disks $\bar{D}_{1, R}(n), \bar{D}_{2, R}(n) \subset V_{n}(t, R)$, with the property that

$$
\left.\widetilde{\Pi}_{t+R+2}\right|_{\bar{D}_{i, R}(n)}: \bar{D}_{i, R}(n) \rightarrow \bar{B}_{\widetilde{M}_{\infty}(t+R+2)}\left(\widetilde{q}_{\infty}, t+R\right) \quad \text { is a } \mu \text {-quasi-isometry, } i=1,2 .
$$

Property (5) implies that $\bar{D}_{i, R}(n)$ can be expressed as the graph of a function

$$
u_{i, n}: \bar{B}_{\widetilde{M}_{\infty}(t+R+2)}\left(\widetilde{q}_{\infty}, t+R\right) \rightarrow \mathbb{R}, \quad i=1,2 .
$$

As the sequence of disks $\left\{\bar{D}_{i, R}(n)\right\}_{n}$ converges as $n \rightarrow \infty$ to $\bar{B}_{\widetilde{M}_{\infty}(t+R+2)}\left(\widetilde{q}_{\infty}, t+R\right)$ for $i=1,2$, then a subsequence of the functions $\frac{u_{1, n}-u_{2, n}}{\left(u_{1, n}-u_{2, n}\right)\left(\widetilde{q}_{\infty}\right)}$ converges as $n \rightarrow \infty$ a non-zero Jacobi function on $\bar{B}_{\widetilde{M}_{\infty}(t+R+2)}\left(\widetilde{q}_{\infty}, t+R\right)$, which has non-zero sign since $\bar{D}_{1, R}(n), \bar{D}_{2, R}(n)$ are disjoint. This contradicts the unstability of $\bar{B}_{\widetilde{M}_{\infty}(t+R+2)}\left(\widetilde{q}_{\infty}, t+R\right)$ and finishes the proof.

Lemma 3.2 For all $R>0$, there exist $t>0$ and $k \in \mathbb{N}$ such that if $m \geq k$, then the component of $\left[\lambda_{m}^{\prime} B_{M}\left(p_{m}^{\prime}, \frac{t}{\lambda_{m}^{\prime}}\right)\right] \cap \overline{\mathbb{B}}(R)$ that passes through $\overrightarrow{0}$ is compact has its boundary on $\mathbb{S}^{2}(R)$.

Proof. We fix $R>0$ and suppose that $M_{\infty}$ intersects transversely the sphere $\mathbb{S}^{2}(R)$ (this transversality property holds for almost every $R$; we will prove the lemma assuming this transversality property, and the lemma will hold for every $R$ after a continuity argument). As $M_{\infty}$ intersects transversely $\mathbb{S}^{2}(R)$, there exists $\varepsilon=\varepsilon(R)>0$ small such that $M_{\infty}$ intersects transversely $\mathbb{S}^{2}\left(R^{\prime}\right)$ for all $R^{\prime} \in[R, R+\varepsilon]$. Given $R^{\prime} \in[R, R+\varepsilon]$, let $\Delta\left(R^{\prime}\right)$ be the component of $M_{\infty} \cap \overline{\mathbb{B}}\left(R^{\prime}\right)$ that contains $\overrightarrow{0}$. Note that $\Delta(R+\varepsilon)$ is compact and contained in the interior of some intrinsic geodesic disk $B_{M_{\infty}}\left(\overrightarrow{0}, R_{1}\right), R_{1}=R_{1}(R) \geq R+\varepsilon$. Also note that $B_{M_{\infty}}\left(\overrightarrow{0}, R_{1}\right)$ is the limit as $n \rightarrow \infty$ of the intrinsic geodesic disks $\lambda_{n}^{\prime} B_{M}\left(p_{n}^{\prime}, \frac{R_{1}}{\lambda_{n}^{\prime}}\right)$.

Applying Lemma 3.1 to $t=R_{1}+1$, we obtain an integer number $k=k(R)$ such that for each $m \geq k$, the surface $\lambda_{m}^{\prime} B_{M}\left(p_{m}^{\prime}, \frac{R_{1}+1}{\lambda_{m}^{\prime}}\right)$ is contained in a small regular neighborhood $U\left(R_{1}+2\right)$ 
of radius $\delta\left(R_{1}+2\right)>0$ of $M_{\infty}\left(R_{1}+2\right)$ and $\lambda_{m}^{\prime} B_{M}\left(p_{m}^{\prime}, \frac{R_{1}+1}{\lambda_{n}^{\prime}}\right)$ is a small normal graph over its projection $W_{m}\left(R_{1}+1\right)$ to $M_{\infty}\left(R_{1}+2\right)$, i.e.,

$$
\left.\left(\Pi_{R_{1}+2}\right)\right|_{\lambda_{m}^{\prime} B_{M}\left(p_{m}^{\prime}, \frac{R_{1}+1}{\lambda_{m}^{\prime}}\right)}: \lambda_{m}^{\prime} B_{M}\left(p_{m}^{\prime}, \frac{R_{1}+1}{\lambda_{m}^{\prime}}\right) \rightarrow W_{m}\left(R_{1}+1\right) \quad \text { is a diffeomorphism, } \forall m \geq k .
$$

Here we are using the same notation as in the proof of Lemma 3.1; therefore, $\Pi_{R_{1}+2}: U\left(R_{1}+2\right) \rightarrow$ $M_{\infty}\left(R_{1}+2\right)$ is the normal projection associated to the regular neighborhood $U\left(R_{1}+2\right)$ of $M_{\infty}\left(R_{1}+2\right)$.

Note that as $\Delta(R+\varepsilon) \subset B_{M_{\infty}}\left(\overrightarrow{0}, R_{1}\right)$, then there exists $k_{1} \geq k$ such that $\Delta(R+\varepsilon) \subset$ $W_{m}\left(R_{1}+1\right), \forall m \geq k_{1}$. As the distance between $\partial B_{M_{\infty}}\left(\overrightarrow{0}, R_{1}\right)$ and $\partial B_{M_{\infty}}\left(\overrightarrow{0}, R_{1}+1\right)$ is 1 , and $W_{m}\left(R_{1}+1\right)$ converges to $B_{M_{\infty}}\left(\overrightarrow{0}, R_{1}+1\right)$ as $m \rightarrow \infty$, then we assume that for $m$ sufficiently large, $B_{M_{\infty}}\left(\overrightarrow{0}, R_{1}\right)$ lies in $W_{m}\left(R_{1}+1\right)$. Since $\Delta(R+\varepsilon) \subset B_{M_{\infty}}\left(\overrightarrow{0}, R_{1}\right)$, then we conclude that $\Delta(R+\varepsilon) \subset W_{m}\left(R_{1}+1\right)$ for all $m$ sufficiently large. This implies that the compact domains

$$
\Omega(m):=\left(\left.\left(\Pi_{R_{1}+2}\right)\right|_{\lambda_{m}^{\prime} B_{M}\left(p_{m}^{\prime}, \frac{R_{1}+1}{\lambda_{m}^{\prime}}\right)}\right)^{-1}[\Delta(R+\varepsilon)] \subset \lambda_{m}^{\prime} B_{M}\left(p_{m}^{\prime}, \frac{R_{1}+1}{\lambda_{m}^{\prime}}\right)
$$

converge smoothly to $\Delta(R+\varepsilon)$ as $m \rightarrow \infty$. In particular, the sequence of boundaries $\partial \Omega(m)$ converge to the boundary of $\Delta(R+\varepsilon)$, which is contained in the sphere $\mathbb{S}^{2}(R+\varepsilon)$. This implies that for $m$ large, $\partial \Omega(m)$ lies outside $\overline{\mathbb{B}}(R)$. Now the lemma follows by taking $t=R_{1}+1$, as the component of $\left[\lambda_{m}^{\prime} B_{M}\left(p_{m}^{\prime}, \frac{t}{\lambda_{m}^{\prime}}\right)\right] \cap \overline{\mathbb{B}}(R)$ passing through $\overrightarrow{0}$ coincides with the component of $\Omega(m) \cap \overline{\mathbb{B}}(R)$ passing through $\overrightarrow{0}$.

Remark 3.3 Note that Lemmas 3.1 and 3.2 remain valid under the hypotheses that $M_{\infty}$ is not flat and the Gaussian curvature of $\left\{\lambda_{n} B_{M}\left(p_{n}, \frac{t}{\lambda_{n}}\right)\right\}_{n}$ is uniformly bounded for each $t>0$ (with the bound depending on $t$ ).

We next finish the proof of Theorem[1.1. Applying Lemma 3.2 to $R_{n}=\lambda_{n}^{\prime}$, we obtain $t(n)>0$ and $k(n) \in \mathbb{N}$ such that if $M_{n}$ denotes the component of $B_{M}\left(p_{k(n)}^{\prime}, \frac{t(n)}{\lambda_{k(n)}^{\prime}}\right) \cap \bar{B}_{N}\left(p_{k(n)}^{\prime}, \frac{\lambda_{n}^{\prime}}{\lambda_{k(n)}^{\prime}}\right)$ that contains $p_{k(n)}^{\prime}$, then $M_{n}$ is compact and its boundary satisfies $\partial M_{n} \subset \partial B_{N}\left(p_{k(n)}^{\prime}, \frac{\lambda_{n}^{\prime}}{\lambda_{k(n)}^{\prime}}\right)$. Clearly this compactness property remains valid if we increase the value of $k(n)$. Hence, we may assume without loss of generality that

$$
t(n)(n+1)<k(n) \text { for all } n, \quad \frac{\lambda_{n}^{\prime}}{\lambda_{k(n)}^{\prime}} \rightarrow 0 \text { as } n \rightarrow \infty .
$$

We now define $p_{n}=p_{k(n)}^{\prime}, \varepsilon_{n}=\frac{\lambda_{n}^{\prime}}{\lambda_{k(n)}^{\prime}}$ and $\lambda_{n}=\lambda_{k(n)}^{\prime}$. Then it is easy to check that the $p_{n}, \varepsilon_{n}, \lambda_{n}$ and $M_{n}$ satisfy the conclusions stated in Theorem 1.1 (in order to prove item 2 in the 
statement of Theorem 1.1, simply note that equations (11) and (2) imply that $\frac{\left|\sigma_{M_{n}}\right|}{\lambda_{n}}=\frac{\left|\sigma_{M_{n}}\right|}{\lambda_{k(n)}^{\prime}} \leq$ $1+\frac{t(n)}{k(n)-t(n)}<1+\frac{1}{n}$, where the last inequality follows from $\left.t(n)(n+1)<k(n)\right)$. This finishes the proof of Theorem 1.1 .

Remark 3.4 If the surface $M \subset N$ in Theorem [1.1 were properly embedded, then the arguments needed to carry out its proof could be formulated in a more standard manner by using the techniques developed in the papers [16, 20]. It is the non-properness of $M$ that necessitates being more careful in defining the limit surface $M_{\infty}$ and in proving additional properties of how it arises as a limit surface of compact embedded minimal surfaces that appear in the blow-up procedure in the proof of Theorem 1.1 .

\section{Applications of Theorem 1.1.}

In any flat three-torus $\mathbb{T}^{3}$, there exists a sequence $\left\{M_{n}\right\}_{n}$ of embedded, compact minimal surfaces of genus three, such that the areas of these surfaces diverge to infinity [11] (a similar result holds for any genus $g \geq 3$, see Traizet [27]). After choosing a subsequence, these surfaces converge to a minimal foliation of $\mathbb{T}^{3}$ and the convergence is smooth away from two points. Since by the GaussBonnet formula, these surfaces have absolute total curvature $8 \pi$, this example demonstrates a special case of Theorem 4.2 below. Before stating this result, we recall a somewhat standard result concerning limits of minimal surfaces. A similar statement can be found in item 5 of Lemma A.1 in Meeks and Rosenberg [21].

Lemma 4.1 Suppose that $\left\{M_{n}\right\}_{n}$ is a sequence of complete embedded minimal surfaces in a Riemannian three-manifold $N$, which converge to minimal lamination $\mathcal{L}$ of $N$. Let $L$ be a leaf of $\mathcal{L}$ which is either a limit leaf of $\mathcal{L}$ or it is an isolated leaf and in this case, the convergence of the sequence $\left\{M_{n}\right\}_{n}$ to $L$ has multiplicity greater than 1. Then, the two-sided cover of $L$ is stable.

Proof. If $L$ is a limit leaf of $\mathcal{L}$, then the main theorem in [19] insures that the two-sided cover of $L$ is stable. Next suppose that $L$ is an isolated leaf of $\mathcal{L}$ and that the convergence of the $M_{n}$ to $L$ has multiplicity greater than 1 . Consider a compact subdomain $D \subset L$ and let $D_{\delta}$ be a regular neighborhood of $D$ in $N$ of small radius $\delta>0$. After possibly lifting to a two-sheeted cover of $D_{\delta}$, we may assume that $D$ is two-sided. Thus $D_{\delta}$ is diffeomorphic to $D \times[-\delta, \delta]$. Since $L$ is isolated as a leaf of $\mathcal{L}$, then the 'top' and 'bottom' sides $D \times\{-\delta\}$ and $D \times\{\delta\}$ of $D_{\delta}$ can be assumed to be disjoint from $\mathcal{L}$ and, since they are compact, they are also disjoint from the surfaces $M_{n}$ for $n$ sufficiently large. Another consequence of the convergence of the $M_{n}$ to $L$ and of the compactness of $D$ is that we may assume that the $M_{n} \cap D_{\delta}$ are locally graphs over their projections to $D$. Consider the sequence of minimal laminations $\left\{\overline{M_{n} \cap D_{\delta}}\right\}_{n}$, which converges to $D$. Note that for $n$ large, each normal unit speed geodesic $\gamma_{x}$ in $D_{\delta}$ starting at a point 
$x \in D$ intersects the lamination $\overline{M_{n} \cap D_{\delta}}$ in a closed set which has a highest point $\gamma_{x}\left(t_{n}(x)\right)$ and a lowest point $\gamma_{x}\left(s_{n}(x)\right)$, for some real numbers $s_{n}(x) \leq t_{n}(x)$. As the multiplicity of the limit $M_{n} \rightarrow L$ is greater than one, then $s_{n}(x)<t_{n}(x)$ for each $n \in \mathbb{N}$. Consider the function $u_{n}(x)=t_{n}(x)-s_{n}(x)$, for all $x \in D$. Since the lamination $\overline{M_{n} \cap D_{\delta}}$ is minimal for each $n \in \mathbb{N}$, then after normalizing $u_{n}$ to be 1 at some point $p \in \operatorname{Int}(D)$, a standard argument shows that these normalized functions converge to a positive Jacobi function of $D$, which implies that $D$ is stable. Finally, $L$ is stable as every compact subdomain $D \subset L$ is stable.

Theorem 4.2 Suppose $\left\{M_{n}\right\}_{n}$ is a sequence of complete, embedded minimal surfaces in a Riemannian three-manifold $N$, such that there exists a open covering of $N$ and $\int_{M_{n} \cap B}\left|\sigma_{n}\right|^{2}$ is uniformly bounded for any open set $B$ in this covering (here $\sigma_{n}$ denotes the second fundamental form of $\left.M_{n}\right)$. Then, there exists a subsequence of $\left\{M_{n}\right\}_{n}$ that converges to a minimal lamination $\mathcal{L}$ of $N$, and the singular set of convergence of the $M_{n}$ to $\mathcal{L}$, defined as

$S(\mathcal{L})=\left\{p \in \mathcal{L} \mid\right.$ the sequence $\left\{\left|\sigma_{M_{n}}\right|\right\}_{n}$ is not uniformly bounded in any neighborhood of $\left.p\right\}$,

is closed and discrete. Furthermore:

1. If $L$ is a limit leaf of $\mathcal{L}$ or a leaf with infinite multiplicity as a limit of the surfaces $M_{n}$, then the two-sided cover of $L$ is stable and $L$ is totally geodesic.

2. If each $M_{n}$ is connected and $N$ is compact, then $\mathcal{L}$ is compact and connected in the subspace topology.

Proof. We will distinguish between good and bad points of $N$, depending on whether or not the surfaces $M_{n}$ have a good behavior around the point to take limits; the set $A \subset N$ of bad points will be then proven to be discrete and closed in $N$, and we will produce a limit minimal lamination $\mathcal{L}$ of the $\left\{M_{n}\right\}_{n}$ in $N-A$. The final step in the proof of the first statement in the theorem will be to show that $\mathcal{L}$ extends as a minimal lamination across $A$.

Let $q$ be a point in $N$. We will say that $q$ is a bad point for the sequence $\left\{M_{n}\right\}_{n}$ if there exists a subsequence $\left\{M_{n_{k}}\right\}_{k} \subset\left\{M_{n}\right\}_{n}$ such that

$$
\int_{M_{n_{k}} \cap B_{N}\left(q, \frac{1}{k}\right)}\left|\sigma_{M_{n_{k}}}\right|^{2} \geq 2 \pi, \quad \text { for all } k \in \mathbb{N} .
$$

First note that we can replace the covering in the statement by a countable open covering of $N$ by balls $B_{i}, i \in \mathbb{N}$. Assume for the moment that $B_{1}$ contains a bad point $q_{1}$ for $\left\{M_{n}\right\}_{n}$. We claim that $B_{1}$ has a finite number of bad points after replacing $\left\{M_{n}\right\}_{n}$ by a subsequence. To see this, since $q_{1}$ is a bad point for $\left\{M_{n}\right\}_{n}$, there exists a subsequence $\left\{M_{k}^{\prime}=M_{n_{k}}\right\}_{k} \subset\left\{M_{n}\right\}_{n}$ such that the total curvature of every $M_{k}^{\prime}$ in $B_{N}\left(q_{1}, \frac{1}{k}\right)$ is at least $2 \pi$. Suppose that $q_{2} \in B_{1}$ is another bad point for $\left\{M_{k}^{\prime}\right\}_{k}$. Then we find a subsequence $\left\{M_{j}^{\prime \prime}=M_{k_{j}}\right\}_{j} \subset\left\{M_{k}^{\prime}\right\}_{k}$ such that 
the total curvature of every $M_{j}^{\prime \prime}$ in $B_{N}\left(q_{2}, \frac{1}{j}\right)$ is at least $2 \pi$. In particular, for $j$ large, there are disjoint neighborhoods of $q_{1}$ and $q_{2}$ in $B_{1}$, each with total curvature of $M_{j}^{\prime \prime}$ at least $2 \pi$. Since $\left\{\int_{M_{n} \cap B_{1}}\left|\sigma_{n}\right|^{2}\right\}_{n}$ is uniformly bounded, this process of finding bad points and subsequences in $B_{1}$ stops after a finite number of steps, which proves our claim. A standard diagonal argument then shows that after replacing the $M_{n}$ by a subsequence, the set of bad points $A \subset N$ for $\left\{M_{n}\right\}_{n}$ is a discrete closed set in $N$.

Suppose that $q \in N-A$. We claim that $\left\{M_{n}\right\}_{n}$ has pointwise bounded second fundamental form in some neighborhood of $q$. Arguing by contradiction, suppose there exist points $p_{n} \in M_{n}$ converging to $q$ and such that $\left|\sigma_{M_{n}}\right|\left(p_{n}\right) \rightarrow \infty$ as $n \rightarrow \infty$. Let $\varepsilon_{q}=\frac{1}{2} d_{N}(q, A)>0$. By the Local Picture Theorem on the Scale of Curvature, there exists a blow-up limit of the $M_{n}$ that converges to a non-flat, properly embedded minimal surface in $\mathbb{R}^{3}$; as the total curvature of a non-flat, complete minimal surface in $\mathbb{R}^{3}$ is at least $4 \pi$, and the $L^{2}$-norm of the second fundamental form is invariant under rescalings of the ambient metric, then we may assume that for $n$ large,

$$
\int_{M_{n} \cap B_{N}\left(q, r_{n}\right)}\left|\sigma_{n}\right|^{2}>2 \pi
$$

where $r_{n} \searrow 0$ satisfies $d_{N}\left(q, p_{n}\right)<r_{n}<\frac{\varepsilon_{q}}{2}$. This clearly contradicts that $q \in N-A$, and so, we conclude that $\left\{M_{n}\right\}_{n}$ has pointwise bounded second fundamental form in some neighborhood $U_{q}$ of $q$. Therefore, there exists a minimal lamination $\mathcal{L}_{q}$ of $U_{q}$ such that a subsequence of the $M_{n}$ converges as $n \rightarrow \infty$ to $\mathcal{L}_{q}$ in $U_{q}$. Another standard diagonal argument proves that after extracting a subsequence, the $M_{n}$ converge to a minimal lamination $\mathcal{L}$ of $N-A$.

Next we show that $\mathcal{L}$ extends across $\mathcal{A}$ to a minimal lamination of $N$. Consider the second fundamental form $\left|\sigma_{\mathcal{L}}\right|$ of $\mathcal{L}$. We claim that $\left|\sigma_{\mathcal{L}}\right|$ does not grow faster than linearly at any point $q \in A$ in terms of the inverse of the extrinsic distance function to $q$ : otherwise, there exists a sequence of blow-up points $p_{n} \in \mathcal{L}$ converging to a point $q \in A$ with $\left|\sigma_{L_{n}}\right|\left(p_{n}\right) d_{N}\left(p_{n}, q\right)$ unbounded, where $L_{n}$ is the leaf of $\mathcal{L}$ passing through $p_{n}$. Using again the Local Picture Theorem on the Scale of Curvature, we deduce that there exist disjoint small neighborhoods $V\left(p_{n}\right)$ of $p_{n}$ in $L_{n}$, such that

$$
\int_{V\left(p_{n}\right)}\left|\sigma_{L_{n}}\right|^{2}>2 \pi, \quad \text { for all } n \in \mathbb{N} .
$$

Since $M_{n}$ converges to $\mathcal{L}$, this contradicts the hypothesis that $\int_{M_{n} \cap B}\left|\sigma_{n}\right|^{2}$ is uniformly bounded for the open set $B$ in the covering which contains $q$. Once we know that $\left|\sigma_{\mathcal{L}}\right|$ does not grow faster than linearly at any point of the discrete closed set $A$, Theorem 2.2 implies that $\mathcal{L}$ extends across $A$ to a minimal lamination of $N$. Observe that by construction, the singular set of convergence $S(\mathcal{L})$ defined in (6) coincides with the set $A$ of bad points. This proves the first sentence of the theorem.

We next prove item 1. Let $L$ be a limit leaf of $\mathcal{L}$. By the main result in [19], the two-sided cover of $L$ is stable. If $L$ is not totally geodesic, then there exists $q \in L-S(\mathcal{L})$ such that $\left|\sigma_{L}\right|(q)=4 \varepsilon>0$ (recall that $S(\mathcal{L})$ is closed and discrete). Then, there exists some open set 
$U \subset[L-S(\mathcal{L})] \cap B$ such that $\left|\sigma_{L}\right| \geq 2 \varepsilon$ in $U$, where $B$ is an open set in the covering that appears in the statement of the theorem, such that $q \in B$. As $L$ is a limit leaf of $\mathcal{L}$ and $L$ is the limit in $N-S(\mathcal{L})$ of the $M_{n}$, then there exist pairwise disjoint domains $U_{n} \subset M_{n} \cap B$ such that $\left|\sigma_{n}\right| \geq \varepsilon$ in $U_{n}$ for all $n$. This clearly contradicts that $\int_{M_{n} \cap B}\left|\sigma_{n}\right|$ is bounded. This proves item 1 of the theorem when $L$ is a limit leaf of $\mathrm{L}$.

If $L$ is not a limit leaf of $\mathcal{L}$ but the multiplicity of the limit $\left\{M_{n}\right\}_{n} \rightarrow L$ is infinity, then Lemma 4.1 insures that the two-sided cover of $L-S(\mathcal{L})$ is stable. Given a compact subdomain $D$ of the two-sided cover $\widehat{L}$ of $L$, the fact that $S(\mathcal{L})$ is closed and discrete implies that $D \cap S(\mathcal{L})$ is finite. After enlarging slightly $D$, we can assume that $D \cap S(\mathcal{L})$ lies in the interior of $D$. As $\widehat{L}-S(\mathcal{L})$ is stable, then $D-S(\mathcal{L})$ is also stable. A standard argument in elliptic theory then shows that $D$ is also stable, and thus $\widehat{L}$ is also stable, as desired. The arguments in the last paragraph to show that $L$ is totally geodesic can be adapted easily to this case, since the multiplicity of the limit $\left\{M_{n}\right\}_{n} \rightarrow L$ is infinity. Now item 1 of the theorem is proved.

The proof of item 2 of the theorem is straightforward and we leave it for the reader.

Remark 4.3 Under the hypotheses and notation of Theorem 4.2, we cannot conclude that $\mathcal{L}$ is path-connected in the subspace topology when $M$ is connected and $N$ is compact: a counterexample can be found for geodesic laminations in Example 3.5 of [18], and this example can be adapted to produce a minimal lamination counterexample simply by taking products with $\mathbb{S}^{1}$.

We now give another application of Theorem 1.1, which is a partial positive answer to Conjecture 1.7 in [17]. Given a two-sided minimal surface $M$ in a flat three-manifold $N$ and given $a>0$, we say that $M$ is a-stable if for any compactly supported smooth function $u \in C_{0}^{\infty}(M)$, we have

$$
\int_{M}\left(|\nabla u|^{2}+a K u^{2}\right) \geq 0
$$

where $\nabla u$ stands for the gradient of $u$ and $K$ is the Gaussian curvature of $M$ (the usual stability condition for the area functional corresponds to the case $a=2$ ). More generally, we say that $M$ has finite index of $a$-stability if there is a bound on the number of negative eigenvalues (counted with multiplicities) of the operator $\Delta-a K$ acting on smooth functions defined on compact subdomains of $M$.

The authors conjectured in [17] (Conjecture 1.7) that if $a>0$ and $M$ is a two-sided, complete, embedded, $a$-stable minimal surface in a complete flat three-manifold $N$, then $M$ is totally geodesic (flat). Do Carmo and Peng [6], Fischer-Colbrie and Schoen [8] and Pogorelov [24] proved independently that if $M$ is a complete, orientable $a$-stable minimal surface immersed in $\mathbb{R}^{3}$, for $a \geq 1$, then $M$ is a plane. This result was later improved by Kawai [10] to $a>1 / 4$, see also Ros [25]. In [17, the authors proved the conjecture (for every $a>0$ and in any complete flat three-manifold $N$ ) under the additional hypotheses that $M$ is embedded and it has finite genus. On the other hand, for small values of $a>0$, there exist complete, non-flat, immersed, 
$a$-stable minimal surfaces in $\mathbb{R}^{3}$ : for instance, apply Lemma 6.3 in [17 to the universal cover of any doubly periodic Scherk minimal surface. In the next result we will prove the conjecture for $a>1 / 8$ assuming solely that $M$ is embedded. It should be also mentioned that FischerColbrie [7] proved item 3 of the next result in the case $a \geq 1$, independently of whether or not $M$ is embedded.

Theorem 4.4 Let $a \in(1 / 8, \infty)$ and $M$ be a two-sided, complete, embedded, minimal surface with compact boundary $\partial M$ in a complete, flat three-manifold $N$. Then:

1. If $\partial M=\varnothing$ and $M$ is a-stable, then $M$ is totally geodesic.

2. There exists $C>0$ (independent of $M, N$ ) such that if $\partial M \neq \varnothing$ and $M$ is a-stable, then $\left|\sigma_{M}\right| d_{M}(\cdot, \partial M) \leq C$ and $M$ has finite total curvature.

3. If $N=\mathbb{R}^{3}$ and $M$ has finite index of a-stability, then $M$ has finite total curvature.

Proof. Suppose that $M \subset N$ has empty boundary and is $a$-stable. After lifting $M$ to $\mathbb{R}^{3}$ and applying Theorem 1.1 (note that $a$-stability is preserved after lifting to a covering, rescaling and taking smooth limits), we can assume that $M$ has bounded Gaussian curvature and $N=\mathbb{R}^{3}$, in particular $M$ is proper [20]. A straightforward application of the maximum principle at infinity implies that $M$ has an embedded regular neighborhood of fixed positive radius and so, $M$ has at most cubical extrinsic area growth, see Meeks and Rosenberg [22]. The following applications of previous results show that $M$ is homeomorphic to $\mathbb{C}$ or to $\mathbb{C}-\{0\}$ :

- If $a>1 / 4$, then apply Theorem 2.9 in [18] (see also Castillon [3]).

- If $a=1 / 4$, then apply part (ii) of Theorem 1.2 in Berard and Castillon [1].

- If $\frac{1}{8}<a<\frac{1}{4}$, then the cubical extrinsic area growth property of $M$ implies that the intrinsic area growth of $M$ is $k_{a}$-subpolynomial, where $k_{a}=2+\frac{4 a}{1-4 a}$. This means that the limit as $r \rightarrow \infty$ of the area of the intrinsic ball in $M$ of radius $r$ (centered at any fixed point) divided by $r^{k_{a}}$ is zero. Now apply part (iii) of Theorem 1.2 in [1].

As $M$ is a properly embedded minimal surface in $\mathbb{R}^{3}$ homeomorphic to $\mathbb{C}$ or to $\mathbb{C}-\{0\}$, then $M$ is either a plane, a catenoid [26] or a helicoid [20]. As both the catenoid and the helicoid are $a$-unstable for all $a>0$ by Proposition 1.5 in [17], we deduce item 1 of the theorem.

Item 2 follows from item 1 by a rescaling argument on the scale of curvature that is given in the proof of Theorem 1.1 (also see the proof of Theorem 15 in [12] for a similar argument).

To see item 3, suppose that $M \subset \mathbb{R}^{3}$ has finite index of $a$-stability. By Proposition 1 in Fischer-Colbrie [7, there exists a compact domain $\Omega \subset M$ such that $M-\operatorname{Int}(\Omega)$ is $a$-stable. In this situation, the already proven item 2 of this theorem implies $M$ has quadratic decay of curvature. Then, Theorem 2.4 implies that $M$ has finite total curvature. 


\section{Proof of the Dynamics Theorem.}

Our next goal is to prove the Dynamics Theorem (Theorem 1.3) stated in the introduction. Regarding the notions introduced in Definition 1.2, we make the following observations.

(i) If $\Sigma \in D(M)$, then $D(\Sigma) \subset D(M)$ (this follows by considering double limits).

This property allows us to consider $D$ as an operator $D: D(M) \rightarrow \mathcal{P}(D(M))$, where $\mathcal{P}(D(M))$ denotes the power set of $D(M)$.

(ii) If $\Sigma \in D(M)$ and $D(\Sigma)=\varnothing$, then $\{\Sigma\}$ is a minimal $D$-invariant set.

(iii) $\Sigma \in D(M)$ is quasi-dilation-periodic if and only if $\Sigma \in D(\Sigma)$.

(iv) Any minimal element $\Sigma \in D(M)$ is contained in a unique minimal $D$-invariant set.

(v) If $\Delta \subset D(M)$ is a minimal $D$-invariant set and $\Sigma \in \Delta$ satisfies $D(\Sigma) \neq \varnothing$, then $D(\Sigma)=\Delta$ (otherwise $D(\Sigma)$ would be a proper non-empty $D$-invariant subset of $\Delta$ ). In particular, $\Sigma$ is quasi-dilation-periodic.

(vi) If $\Delta \subset D(M)$ is a $D$-invariant set and $\Sigma \in \Delta$ is a minimal element, then the unique minimal $D$-invariant subset $\Delta^{\prime}$ of $D(M)$ which contains $\Sigma$ satisfies $\Delta^{\prime} \subset \Delta$ (otherwise $\Delta^{\prime} \cap \Delta$ would be a proper non-empty $D$-invariant subset of $\Delta^{\prime}$ ).

Proof of Theorem 1.3. First assume that $M$ has finite total curvature. Then, its total curvature outside of some ball in space is less than $2 \pi$, and so, any $\Sigma \in D(M)$ must have total curvature less than $2 \pi$, which implies $\Sigma$ is flat. This gives that $D(M)=\varnothing$.

Reciprocally, assume that $D(M)=\varnothing$ and $M$ does not have finite total curvature. By Theorem 2.4. $M$ does not have quadratic decay of curvature, and so, there exists a divergent sequence of points $z_{n} \in M$ with $\left|K_{M}\right|\left(z_{n}\right)\left|z_{n}\right|^{2} \rightarrow \infty$. Let $p_{n} \in \mathbb{B}\left(z_{n}, \frac{\left|z_{n}\right|}{2}\right)$ be a maximum of the function $h_{n}=\left|K_{M}\right| d_{\mathbb{R}^{3}}\left(\cdot, \partial \mathbb{B}\left(z_{n}, \frac{\left|z_{n}\right|}{2}\right)\right)^{2}$. Note that $\left\{p_{n}\right\}_{n}$ diverges in $\mathbb{R}^{3}$ (because $\left|p_{n}\right| \geq \frac{\left|z_{n}\right|}{2}$ ). We define $\lambda_{n}=\sqrt{\left|K_{M}\right|\left(p_{n}\right)}$. By similar arguments as those in the proof of Theorem 1.1 applied to $M \cap \mathbb{B}\left(p_{n}, \frac{\sqrt{h_{n}\left(p_{n}\right)}}{2 \lambda_{n}}\right)$, the sequence $\left\{\lambda_{n}\left(M-p_{n}\right)\right\}_{n}$ converges (after passing to a subsequence) to a minimal lamination $\mathcal{L}$ of $\mathbb{R}^{3}$ with a non-flat leaf $L$ which passes through $\overrightarrow{0}$ and satisfies $\left|K_{L}\right|(\overrightarrow{0})=1$. Furthermore, the curvature function $K_{\mathcal{L}}$ of $\mathcal{L}$ satisfies $\left|K_{\mathcal{L}}\right| \leq 1$ and so, the leaf $L$ of $\mathcal{L}$ passing through $\overrightarrow{0}$ is properly embedded in $\mathbb{R}^{3}$, as are all the leaves of $\mathcal{L}$; see [22] for this properness result. By the Strong Half-space Theorem, $\mathcal{L}$ consists just of $L$, and the convergence of the surfaces $\lambda_{n}\left(M-p_{n}\right)$ to $L$ has multiplicity one (by Lemma 4.1 since the two-sided cover of $L$ is not stable as $L$ is not flat). Therefore, $L \in D_{1}(M)$, which contradicts that $D(M)=\varnothing$. This proves the equivalence stated in Theorem 1.3 . 
Assume now that $D(M) \neq \varnothing$. Hence, $M$ does not have finite total curvature and the arguments in the last paragraph show that $D_{1}(M) \neq \varnothing$. To conclude the proof of item 1 of the theorem it remains to analyze the topology of $D_{1}(M)$.

We will now define a metric space structure on $D_{1}(M)$ which generates a topology that coincides with the topology of uniform $C^{k}$-convergence on compact subsets of $\mathbb{R}^{3}$ for any $k \in \mathbb{N}$ (in particular, compactness of $D_{1}(M)$ will follow from sequential compactness). To do this, we first prove that there exists some $\varepsilon>0$ such that $\bar{B}(\varepsilon)$ intersects every surface $\Sigma \in D_{1}(M)$ in a unique component which is a graphical disk over its projection to the tangent space to $\Sigma$ at $\overrightarrow{0}$ and with gradient less than 1 . Otherwise, there exists a sequence $\left\{\Sigma_{n}\right\}_{n} \subset D_{1}(M)$ such that this property fails in the ball $\overline{\mathbb{B}}\left(\frac{1}{n}\right)$ for every $n \in \mathbb{N}$. As the Gaussian curvature of $\Sigma_{n}$ is not greater than 1, the uniform graph lemma [23] implies that around every point $p_{n} \in \Sigma_{n}$, this surface can be locally expressed as a graph over a disk in the tangent space $T_{p_{n}} \Sigma_{n}$ of uniform radius. Therefore, there exists $\delta>0$ such that for $n$ large, $\Sigma_{n}$ intersects $\overline{\mathbb{B}}(\delta)$ in at least two components, one of which passes through $\overrightarrow{0}$ and the other one intersects $\mathbb{B}\left(\frac{1}{n}\right)$, and such that both components are graphical over domains in the tangent space of $\Sigma_{n}$ at $\overrightarrow{0}$ with small gradient. Hence, a subsequence of these $\Sigma_{n}$ (denoted in the same way) converges smoothly to a minimal lamination $\mathcal{L}_{1}$ of $\mathbb{R}^{3}$ with a leaf $L_{1} \in \mathcal{L}_{1}$ passing through $\overrightarrow{0}$ such that the multiplicity of the limit $\left\{\Sigma_{n}\right\}_{n} \rightarrow L_{1}$ is greater than one. This last property implies that the two-sided cover $\widehat{L}_{1}$ of $L_{1}$ is stable, by Lemma 4.1. As $\widehat{L}_{1}$ is complete and stable then $\widehat{L}_{1}$ is a plane (and $L_{1}=\widehat{L}_{1}$ ), which contradicts that the convergence $\left\{\Sigma_{n}\right\}_{n} \rightarrow L_{1}$ is smooth and the curvature of the $\Sigma_{n}$ is -1 at $\overrightarrow{0}$ for every $n$. This proves our claim on the existence of $\varepsilon$.

With the above $\varepsilon>0$ at hand, we define the distance between any two surfaces $\Sigma_{1}, \Sigma_{2} \in$ $D_{1}(M)$ as

$$
d\left(\Sigma_{1}, \Sigma_{2}\right)=d_{\mathcal{H}}\left(\Sigma_{1} \cap \overline{\mathbb{B}}(\varepsilon / 2), \Sigma_{2} \cap \overline{\mathbb{B}}(\varepsilon / 2)\right),
$$

where $d_{\mathcal{H}}$ denotes the Hausdorff distance. Standard elliptic theory implies that the metric topology on $D_{1}(M)$ associated to the distance $d$ agrees with the topology of the uniform $C^{k}$ convergence on compact sets of $\mathbb{R}^{3}$ for any $k$.

Next we prove that $D_{1}(M)$ is sequentially compact (hence compact). Every sequence $\left\{\Sigma_{n}\right\}_{n} \subset D_{1}(M)$ contains a subsequence which converges to a minimal lamination $\mathcal{L}$ of $\mathbb{R}^{3}$ with bounded Gaussian curvature $K_{\mathcal{L}}$ and $K_{\mathcal{L}}(\overrightarrow{0})=-1$. The same arguments given in the second paragraph of this proof imply that $\mathcal{L}$ consists just of the leaf $L$ passing through $\overrightarrow{0}$, which is a properly embedded minimal surface in $\mathbb{R}^{3}$. Clearly $L \in D_{1}(M)$, which proves item 1 of the theorem.

We now prove item 2. Using the definition of $D$-invariance, it is elementary to show that for any $\Sigma \in D(M), D(\Sigma)$ is a closed set in $D(M)$; essentially, this is because the set of limit points of a set in a topological space forms a closed set. That $D(\Sigma)$ is $D$-invariant follows from property (i) stated at the beginning of this section. Similar techniques show that if $\Delta \subset D(M)$ is a $D$-invariant subset, then its closure in $D(M)$ is also $D$-invariant, and item 2 of the theorem is proved. 
Now assume that $\Delta$ is a minimal $D$-invariant set in $D(M)$. If $\Delta$ contains a surface of finite total curvature, then the minimality of $\Delta$ implies $\Delta$ consists only of this surface, and so, it is connected and closed in $D(M)$. Suppose now that $\Delta$ does not consist of exactly one surface of finite total curvature, or equivalently, $\Delta$ contains no surfaces of finite total curvature. Then, property (v) above implies that for any $\Sigma \in \Delta, D(\Sigma)=\Delta$. Since $D(\Sigma)$ is closed by item 2, then $\Delta$ is closed as well. Since $D(\Sigma)=\Delta$, then $\Delta$ also contains the path connected subset $S \subset D(M)$ of all dilations of $\Sigma$. Since $\Delta$ is a closed set in $D(M)$, then the closure of $S$ in $D(M)$ is contained in $\Delta$. Reciprocally, if $\Sigma_{1} \in \Delta=D(\Sigma)$ then $\Sigma_{1}$ is a non-flat, properly embedded minimal surface in $\mathbb{R}^{3}$ which is the $C^{2}$-limit of a sequence $\mu_{n}\left(\Sigma-p_{n}\right)$ for some $\left\{\mu_{n}\right\}_{n} \subset \mathbb{R}^{+}$and $\left\{p_{n}\right\}_{n} \subset \mathbb{R}^{3}, p_{n} \rightarrow \infty$. As $\mu_{n}\left(\Sigma-p_{n}\right) \in S$ for each $n \in \mathbb{N}$, then $\Sigma_{1}$ lies in the closure of $S$ in $D(M)$, and so, $\Delta$ equals the closure of $S$ in $D(M)$ (in particular, $\Delta$ is connected as $S$ is path connected). This proves item 3 of the theorem.

Next we prove item 4 . Suppose $\Delta \subset D(M)$ is a non-empty, $D$-invariant set. One possibility is that $\Delta$ contains a surface $\Sigma$ of finite total curvature. By the main statement of this theorem, $D(\Sigma)=\varnothing$ and by property (ii) above, $\Sigma$ is a minimal element in $\Delta$. Now assume $\Delta$ contains no surfaces of finite total curvature. Consider the collection

$$
\Lambda=\left\{\Delta^{\prime} \subset \Delta \mid \Delta^{\prime} \text { is non-empty, closed and } D \text {-invariant }\right\}
$$

Note that $\Lambda$ is non-empty, since for any $\Sigma \in \Delta$, the set $D(\Sigma) \subset \Delta$ is such a closed, non-empty $D$-invariant set by the first statement in item 2. $\Lambda$ has a partial ordering induced by inclusion. We just need to check that any linearly ordered subset in $\Lambda$ has a lower bound, and then apply Zorn's Lemma to obtain item 4 of the theorem. Suppose $\Lambda^{\prime} \subset \Lambda$ is a non-empty linearly ordered subset. We must check that the intersection $\bigcap_{\Delta^{\prime} \in \Lambda^{\prime}} \Delta^{\prime}$ is an element of $\Lambda$. In our case, this means we only need to prove that such an intersection is non-empty, because the intersection of closed (resp. $D$-invariant) sets is closed (resp. $D$-invariant).

Given $\Delta^{\prime} \in \Lambda^{\prime}$, consider the collection of surfaces $\Delta_{1}^{\prime}=\left\{\Sigma \in \Delta^{\prime}|\overrightarrow{0} \in \Sigma,| K_{\Sigma}|\leq 1,| K_{\Sigma} \mid(\overrightarrow{0})=\right.$ 1\}. Note that $\Delta_{1}^{\prime}$ is a closed subset of $D(M)$, since $\Delta^{\prime}$ and $D_{1}(M)$ are closed in $D(M)$. The set $\Delta_{1}^{\prime}$ is non-empty by the following argument. Let $\Sigma \in \Delta^{\prime}$. Since $\Sigma$ does not have finite total curvature and $\Delta^{\prime}$ is $D$-invariant, $D(\Sigma)$ is a non-empty subset of $\Delta^{\prime}$. By item $1, D_{1}(\Sigma)$ is a non-empty subset of $\Delta_{1}^{\prime}$, and so, $\Delta_{1}^{\prime}$ is non-empty. Now define $\Lambda_{1}^{\prime}=\left\{\Delta_{1}^{\prime} \mid \Delta^{\prime} \in \Lambda^{\prime}\right\}$. As $\bigcap_{\Delta_{1}^{\prime} \in \Lambda_{1}^{\prime}} \Delta_{1}^{\prime}=\bigcap_{\Delta^{\prime} \in \Lambda^{\prime}} \Delta_{1}^{\prime}=\bigcap_{\Delta^{\prime} \in \Lambda^{\prime}}\left(\Delta^{\prime} \cap D_{1}(M)\right)=\left(\bigcap_{\Delta^{\prime} \in \Lambda^{\prime}} \Delta^{\prime}\right) \cap D_{1}(M)$, in order to check that $\bigcap_{\Delta^{\prime} \in \Lambda^{\prime}} \Delta^{\prime}$ is non-empty, it suffices to show that $\bigcap_{\Delta_{1}^{\prime} \in \Lambda_{1}^{\prime}} \Delta_{1}^{\prime}$ is non-empty. But this is clear since each element of $\Lambda_{1}^{\prime}$ is a closed subset of the compact metric space $D_{1}(M)$, and the finite intersection property holds for the collection $\Lambda_{1}^{\prime}$.

Next we prove item 5 . Let $\Delta \subset D(M)$ be a non-empty $D$-invariant subset which contains no surfaces of finite total curvature. By item 4 , there exists a minimal element $\Sigma \in \Delta$. Since none of the surfaces of $\Delta$ have finite total curvature, it follows that $D(\Sigma) \neq \varnothing$. As $\Sigma$ is a minimal element, there exists a minimal $D$-invariant subset $\Delta^{\prime} \subset D(M)$ such that $\Sigma \in \Delta^{\prime}$. By property (v) above, $D(\Sigma)=\Delta^{\prime}$. Note that $\Delta_{1}^{\prime}=\Delta^{\prime} \cap D_{1}(M)$ contains $D_{1}(\Sigma)$, which is 
non-empty since $D(\Sigma) \neq \varnothing$ (by item 1 of this theorem). Then there exists a surface $\Sigma_{1} \in \Delta_{1}^{\prime}$, which in particular is a minimal element (any element of $\Delta^{\prime}$ is), and lies in $\Delta_{1}$ (because $\Delta^{\prime} \subset \Delta$ by property $(\mathbf{v i})$ ). Finally, $\Sigma_{1}$ is dilation-periodic by property $(\mathbf{v})$, thereby proving item 5 of the theorem.

To prove item 6, suppose $\Sigma \in D(M)$ is a minimal element with finite genus, and assume also that $\Sigma$ has infinite total curvature. Since $\Sigma \in D(\Sigma)$ (by property $(\mathbf{v})$ applied to $\Delta=D(\Sigma)$ ) and $\Sigma$ has finite genus, then the genus of $\Sigma$ must be zero. In this setting, the classification results in [4, 20, 15] imply that $\Sigma$ is a helicoid or a Riemann minimal example.

Finally we prove item 7 . Consider a minimal element $\Sigma$ of $D(M)$ with more than one end. By the Ordering Theorem [9], after possibly a rotation in $\mathbb{R}^{3}$ so that the limit tangent plane at infinity for $\Sigma$ is horizontal (see [2] for this notion of limit tangent plane at infinity), $\Sigma$ has a middle end $e$ in the natural ordering of the ends of $\Sigma$ by their relative heights. The results in [5] imply that $e$ is a simple end and $e$ admits an end representative $E \subset \Sigma$ with the following properties:

- $E$ is a proper, non-compact subdomain of $\Sigma$ with compact boundary and only one end.

- $E$ is contained in the open region $W \subset \mathbb{R}^{3}-\mathbb{B}(R)$ between two graphical, disjoint vertical half-catenoids or horizontal planes, where $R>0$ is sufficiently large. Furthermore, we can assume that $\Sigma \cap W=E$.

We now discuss two cases for $E$. First assume that $E$ has quadratic decay of curvature. By Theorem 2.4. $E$ has finite total curvature, in which case item 7 is known to hold. So, assume that $E$ has infinite total curvature and we will find a contradiction. On one hand, the proof of the first statement of this theorem applies to $E$ to produce the collection $D(E)$ of properly embedded minimal surfaces in $\mathbb{R}^{3}$ which are limits of $E$ under a sequence of dilations with divergent translational part (note that surfaces in $D(E)$ have empty boundary). Notice that under every divergent sequence of dilations which give rise to a surface in $D(E)$, the dilated regions $W_{n}$ related to $W$ converge to all of $\mathbb{R}^{3}$, by the Half-space Theorem. This implies that $D(E)$ is a subset of $D(\Sigma)$. Since $\Sigma$ is a minimal element, then $D(E)=D(\Sigma)$. On the other hand, the results in [5] imply that $E$ has quadratic area growth, and hence, the monotonicity formula gives that every surface in $D(E)$ has at most the same quadratic area growth as $E$. This discussion applies to $\Sigma$ since $\Sigma \in D(\Sigma)$. But since $\Sigma$ has other ends different from $E$, the quadratic area growth of $\Sigma$ is strictly greater than the one of $E$. This contradiction proves item 7 , thereby finishing the proof of Theorem 1.3 . 


\section{Internal dynamical periodicity of properly embedded minimal surfaces with infinite total curvature.}

Let $M \subset \mathbb{R}^{3}$ be a properly embedded minimal surface with infinite total curvature and let $\Sigma \in D(M)$ be a minimal element, which exists by item 4 of Theorem 1.3. Assume that $\Sigma$ also has infinite total curvature. We claim that each compact subdomain of $\Sigma$ can be approximated with arbitrarily high precision (under dilation) by an infinite collection of pairwise disjoint compact subdomains of $\Sigma$, and these approximations can be chosen not too far from each other. This property can be considered to be a weak notion of periodicity; next we describe a more precise statement for this 'weak periodicity' property for $\Sigma$.

As $\Sigma \in D(M)$ is a minimal element, there exists a minimal $D$-invariant subset $\Delta \subset D(M)$ such that $\Sigma \in \Delta$. Since $\Sigma$ has infinite total curvature, then $D(\Sigma)=\Delta$, in particular $\Sigma \in D(\Sigma)$ (i.e., $\Sigma$ is quasi-dilation-periodic). Given $R>0$ such that $\Sigma$ intersects $\mathbb{S}^{2}(R)$ transversely, let $\Sigma(R)=\Sigma \cap \overline{\mathbb{B}}(R)$. Since $\Sigma \in D(\Sigma)$, then for every small $\varepsilon>0$ there exists a collection $\left\{\overline{\mathbb{B}}_{n}=\overline{\mathbb{B}}\left(p_{n}, R_{n}\right)\right\}_{n}$ of disjoint closed balls such that the surfaces $\Sigma_{n}=\frac{R}{R_{n}}\left(\left(\Sigma \cap \overline{\mathbb{B}}_{n}\right)-p_{n}\right)$ can be parameterized by $\Sigma(R)$ in such a way that as mappings, they are $\varepsilon$-close to $\Sigma(R)$ in the $C^{2}$ norm. By Zorn's lemma, any such collection of balls $\left\{\overline{\mathbb{B}}_{n}\right\}_{n}$ is contained in a maximal collection $\operatorname{Max}(\Sigma, R, \varepsilon)$ of closed round balls $\overline{\mathbb{B}}=\overline{\mathbb{B}}(p, r)$ so that $\frac{R}{r}[(\Sigma \cap \overline{\mathbb{B}})-p]$ can be parameterized by $\Sigma(R)$ in such a way that as mappings, they are $\varepsilon$-close in the $C^{2}$-norm. After denoting the elements of $\operatorname{Max}(\Sigma, R, \varepsilon)$ by $\overline{\mathbb{B}}_{n}, n \in \mathbb{N}$, we define for every $n \in \mathbb{N}$ the positive number

$$
d(n ; R, \varepsilon)=\inf _{m \neq n}\left\{\frac{1}{R_{n}} \operatorname{dist}_{\mathbb{R}^{3}}\left(\overline{\mathbb{B}}_{n}, \overline{\mathbb{B}}_{m}\right) \mid \overline{\mathbb{B}}_{m} \in \operatorname{Max}(\Sigma, R, \varepsilon), m \neq n\right\},
$$

where $\overline{\mathbb{B}}_{n}, \overline{\mathbb{B}}_{m} \in \operatorname{Max}(\Sigma, R, \varepsilon)$. Hence, $d(n ; R, \varepsilon)$ measures the minimum relative distance from the ball $\overline{\mathbb{B}}_{n} \in \operatorname{Max}(\Sigma, R, \varepsilon)$ to any other ball $\overline{\mathbb{B}}_{m}$ in the collection $\operatorname{Max}(\Sigma, R, \varepsilon)$, where by relative we mean that $\overline{\mathbb{B}}_{n}$ is normalized to have radius 1 ; this is the task of dividing by $R_{n}$ in (8). In this situation we will prove the following property, which expresses in a precise way the 'weak periodicity' mentioned in the first paragraph of this section.

Proposition 6.1 If $\Sigma(R)$ is not an $\frac{\varepsilon}{2}$-graph over a disk in a plane, then the sequence $\{d(n ; R, \varepsilon)\}_{n} \subset(0, \infty)$ is bounded from above (the bound depends on $\Sigma, R, \varepsilon$ ).

Proof. We argue by contradiction. Otherwise, there exists a sequence of integers $\{n(i)\}_{i \in \mathbb{N}}$ such that $d(n(i) ; R, \varepsilon) \geq i$ for all $i \in \mathbb{N}$. We define $\widehat{\Sigma}(i)=\frac{R}{R_{n(i)}}\left(\Sigma-p_{n(i)}\right), i \in \mathbb{N}$. Observe that the following two properties hold:

(P1) The Gaussian curvature of $\{\widehat{\Sigma}(i) \cap \overline{\mathbb{B}}(R)\}_{i}$ is uniformly bounded (by the defining properties of the balls in the family $\operatorname{Max}(\Sigma, R, \varepsilon))$.

(P2) Given $\overline{\mathbb{B}}_{m} \in \operatorname{Max}(\Sigma, R, \varepsilon)$ with $m \neq n(i)$, the closed ball $\overline{\mathbb{B}}_{m}^{\prime}=\frac{R}{R_{n(i)}}\left(\overline{\mathbb{B}}_{m}-p_{n(i)}\right)$ is at distance at least $i R$ from $\overline{\mathbb{B}}(R)$. 
We claim that the sequence of surfaces $\widehat{\Sigma}(i)$ has locally bounded Gaussian curvature outside of $\overline{\mathbb{B}}(R)$ : otherwise, the Gaussian curvature $K_{\widehat{\Sigma}(i)}$ blows-up around a point $P \in \mathbb{R}^{3}-\overline{\mathbb{B}}(R)$, and in this case we can blow-up $\widehat{\Sigma}(i)$ on the scale of curvature around points of almost-maximal curvature tending to $P$, thereby obtaining a new limit surface $\Sigma^{\prime}$ of rescaled copies of portions of $\widehat{\Sigma}(i)$ in small extrinsic balls around $P$ (in particular, these small balls are disjoint from the balls corresponding to elements in $\operatorname{Max}(\Sigma, R, \varepsilon)$ ). Hence, $\Sigma^{\prime}$ lies in $D(\Sigma)$; since $\Sigma$ is a minimal element, then $\Sigma \in D\left(\Sigma^{\prime}\right)$, which contradicts the maximality of the collection $\operatorname{Max}(\Sigma, R, \varepsilon)$. This contradiction proves our claim.

As $\left\{\widehat{\Sigma}(i) \cap\left[\mathbb{R}^{3}-\overline{\mathbb{B}}(R)\right]\right\}_{i}$ has locally bounded Gaussian curvature, we conclude that after replacing by a subsequence, $\left\{\widehat{\Sigma}(i) \cap\left[\mathbb{R}^{3}-\overline{\mathbb{B}}(2 R)\right]\right\}_{i}$ converges as $n \rightarrow \infty$ to a minimal lamination $\mathcal{L}^{\prime}(2 R)$ of $\mathbb{R}^{3}-\overline{\mathbb{B}}(2 R)$. This lamination $\mathcal{L}^{\prime}(2 R)$ has quadratic decay of curvature (otherwise we again contradict the minimality of $\Sigma$ and the maximality of $\operatorname{Max}(\Sigma, R, \varepsilon)$ as before), and so, $\mathcal{L}^{\prime}(2 R)$ has bounded curvature in $\mathbb{R}^{3}-\mathbb{B}(3 R)$.

Next we claim that the surfaces $\widehat{\Sigma}(i) \cap \bar{B}(3 R)$ do not have uniformly bounded curvature. Arguing again by contradiction, the failure of our claim together with the arguments in the last paragraph imply that $\{\widehat{\Sigma}(i)\}_{i}$ converges to a lamination $\mathcal{L}^{\prime}$ of $\mathbb{R}^{3}$ with quadratic curvature decay. Since we are assuming that $\Sigma(R)$ is not an $\frac{\varepsilon}{2}$-graph over a flat disk, then $\mathcal{L}^{\prime}$ cannot be flat. Hence, Proposition 2.5 implies that $\mathcal{L}^{\prime}$ consists of a single leaf which is a properly embedded minimal surface $\Sigma^{\prime}$ with finite total curvature. As before, this implies that $\Sigma^{\prime} \in D(\Sigma)$. This is absurd, since then $\Sigma \in D\left(\Sigma^{\prime}\right)=\varnothing$. This contradiction shows that the $\widehat{\Sigma}(i) \cap \bar{B}(3 R)$ do not have uniformly bounded curvature.

Therefore, after extracting a subsequence we can find for each $i \in \mathbb{N}$ a point $\widehat{p}(i) \in \widehat{\Sigma}(i) \cap$ $\overline{\mathbb{B}}(3 R)$ such that $\left|K_{\widehat{\Sigma}(i)}\right|(\widehat{p}(i)) \rightarrow \infty$ as $i \rightarrow \infty$. We can also assume that $\left|K_{\widehat{\Sigma}(i)}\right|$ attains its maximum value in the compact set $\widehat{\Sigma}(i) \cap \overline{\mathbb{B}}(3 R)$ at $\widehat{p}(i)$, for all $i$. After translating by $-\widehat{p}(i)$, rescaling by $\sqrt{\left|K_{\widehat{\Sigma}(i)}\right|(\widehat{p}(i))}$ and extracting another subsequence, we obtain a new limit surface $\Sigma^{\prime \prime} \in D_{1}(\Sigma)$. We now have two possibilities, depending on whether or not the sequence of open balls $\left\{\sqrt{\left|K_{\widehat{\Sigma}(i)}\right|(\widehat{p}(i))}(\mathbb{B}(R)-\widehat{p}(i))\right\}_{i}$ eventually leaves every compact set of $\mathbb{R}^{3}$.

Firstly suppose that the sequence of open balls $\left\{\sqrt{\left|K_{\widehat{\Sigma}(i)}\right|(\widehat{p}(i))}(\mathbb{B}(R)-\widehat{p}(i))\right\}_{i}$ fails to leave every compact set of $\mathbb{R}^{3}$. Then, after choosing a subsequence these balls converge to a closed halfspace $H^{+}$and by property (P1) above, $\Sigma^{\prime \prime} \cap H^{+}$is flat. As $\Sigma^{\prime \prime}$ is not flat, then we conclude that $\Sigma^{\prime \prime}$ cannot intersect $H^{+}$, or equivalently $\Sigma^{\prime \prime}$ is contained in a halfspace. This contradicts the fact that the Gaussian curvature of the non-flat surface $\Sigma^{\prime \prime}$ is bounded, see [20]. Therefore, the sequence of balls $\left\{\sqrt{\left|K_{\widehat{\Sigma}(i)}\right|(\widehat{p}(i))}(\mathbb{B}(R)-\widehat{p}(i))\right\}_{i}$ leaves every compact set of $\mathbb{R}^{3}$. Since $\Sigma^{\prime \prime} \in D(\Sigma)$ and $\Sigma$ is a minimal element, then $\Sigma \in D\left(\Sigma^{\prime \prime}\right)$, which by the same arguments as before contradicts the maximality of the collection $\operatorname{Max}(\Sigma, R, \varepsilon)$. Now the proposition is proved. 
Remark 6.2 We remark that in general there exist properly embedded minimal surfaces $M$ with infinite total curvature, such that $D(M)$ contains more than one minimal $D$-invariant set, see some examples in the discussion before Theorem 11.0.13 in [13].

William H. Meeks, III at bill@math.umass.edu

Mathematics Department, University of Massachusetts, Amherst, MA 01003

Joaquín Pérez at jperez@ugr.es_ Antonio Ros at aros@ugr.es

Department of Geometry and Topology, University of Granada, Granada, Spain

\section{References}

[1] P. Bérard and P. Castillon. Inverse spectral positivity for surfaces. Preprint, arXiv: 1111.5928v3, 2011.

[2] M. Callahan, D. Hoffman, and W. H. Meeks III. The structure of singly-periodic minimal surfaces. Invent. Math., 99:455-481, 1990. MR1032877, Zbl 695.53005.

[3] P. Castillon. An inverse spectral problem on surfaces. Comment. Math. Helv., 81(2):271286, 2006. MR2225628 (2007b:58042), Zbl 1114.58025.

[4] P. Collin. Topologie et courbure des surfaces minimales de $\mathbb{R}^{3}$. Ann. of Math. (2), 1451:1-31, 1997. MR1432035, Zbl 886.53008.

[5] P. Collin, R. Kusner, W. H. Meeks III, and H. Rosenberg. The geometry, conformal structure and topology of minimal surfaces with infinite topology. J. Differential Geom., 67:377-393, 2004. MR2153082, Zbl 1098.53006.

[6] M. do Carmo and C. K. Peng. Stable complete minimal surfaces in $\mathbb{R}^{3}$ are planes. Bulletin of the AMS, 1:903-906, 1979. MR0546314, Zbl 442.53013.

[7] D. Fischer-Colbrie. On complete minimal surfaces with finite Morse index in 3-manifolds. Invent. Math., 82:121-132, 1985. MR0808112, Zbl 0573.53038.

[8] D. Fischer-Colbrie and R. Schoen. The structure of complete stable minimal surfaces in 3-manifolds of nonnegative scalar curvature. Comm. on Pure and Appl. Math., 33:199-211, 1980. MR0562550, Zbl 439.53060.

[9] C. Frohman and W. H. Meeks III. The ordering theorem for the ends of properly embedded minimal surfaces. Topology, 36(3):605-617, 1997. MR1422427, Zbl 878.53008.

[10] S. Kawai. Operator $\Delta-a K$ on surfaces. Hokkaido Math. J., 17(2):147-150, 1988. MR0945852 (89j:58149), Zbl 0653.53044. 
[11] W. H. Meeks III. The theory of triply-periodic minimal surfaces. Indiana Univ. Math. J., 39(3):877-936, 1990. MR1078743, Zbl 721.53057.

[12] W. H. Meeks III and J. Pérez. Conformal properties in classical minimal surface theory. In Surveys of Differential Geometry IX - Eigenvalues of Laplacian and other geometric operators, pages 275-336. International Press, edited by Alexander Grigor'yan and Shing Tung Yau, 2004. MR2195411, Zbl 1086.53007.

[13] W. H. Meeks III and J. Pérez. A survey on classical minimal surface theory, volume 60 of University Lecture Series. AMS, 2012. ISBN: 978-0-82; Preprint available at http://www.ugr.es/local/jperez/papers/papers.htm.

[14] W. H. Meeks III, J. Pérez, and A. Ros. Local removable singularity theorems for minimal laminations. Preprint available at http://www.ugr.es/local/jperez/papers/papers.htm.

[15] W. H. Meeks III, J. Pérez, and A. Ros. Properly embedded minimal planar domains. Preprint available at http://www.ugr.es/local/jperez/papers/papers.htm.

[16] W. H. Meeks III, J. Pérez, and A. Ros. The geometry of minimal surfaces of finite genus I; curvature estimates and quasiperiodicity. J. Differential Geom., 66:1-45, 2004. MR2128712, Zbl 1068.53012.

[17] W. H. Meeks III, J. Pérez, and A. Ros. Liouville-type properties for embedded minimal surfaces. Communications in Analysis and Geometry, 14(4):703-723, 2006. MR2273291, Zbl 1117.53009.

[18] W. H. Meeks III, J. Pérez, and A. Ros. Stable constant mean curvature surfaces. In Handbook of Geometrical Analysis, volume 1, pages 301-380. International Press, edited by Lizhen Ji, Peter Li, Richard Schoen and Leon Simon, ISBN: 978-1-57146-130-8, 2008. MR2483369, Zbl 1154.53009.

[19] W. H. Meeks III, J. Pérez, and A. Ros. Limit leaves of an H lamination are stable. J. Differential Geometry, 84(1):179-189, 2010. MR2629513, Zbl 1197.53037.

[20] W. H. Meeks III and H. Rosenberg. The uniqueness of the helicoid. Ann. of Math., 161:723-754, 2005. MR2153399, Zbl 1102.53005.

[21] W. H. Meeks III and H. Rosenberg. The minimal lamination closure theorem. Duke Math. Journal, 133(3):467-497, 2006. MR2228460, Zbl 1098.53007.

[22] W. H. Meeks III and H. Rosenberg. Maximum principles at infinity. J. Differential Geometry, 79(1):141-165, 2008. MR2401421, Zbl pre05285650. 
[23] J. Pérez and A. Ros. Properly embedded minimal surfaces with finite total curvature. In The Global Theory of Minimal Surfaces in Flat Spaces, pages 15-66. Lecture Notes in Math 1775, Springer-Verlag, 2002. G. P. Pirola, editor. MR1901613, Zbl 1028.53005.

[24] A. V. Pogorelov. On the stability of minimal surfaces. Soviet Math. Dokl., 24:274-276, 1981. MR0630142, Zbl 0495.53005.

[25] A. Ros. The Gauss map of minimal surfaces. In O. Gil-Medrano and V. Miquel, editors, Differential Geometry, Valencia 2001, Proceedings of the conference in honour of Antonio M. Naveira, pages 235-252. World Scientific, 2002. MR1922054, Zbl 1028.53008.

[26] R. Schoen. Uniqueness, symmetry, and embeddedness of minimal surfaces. J. Differential Geom., 18:791-809, 1983. MR0730928, Zbl 0575.53037.

[27] M. Traizet. On the genus of triply periodic minimal surfaces. J. Differential Geom., 79:243-275, 2008. MR2420019, Zbl pre05294962. 